\title{
MetaTel: Ongoing work on a meta-material sub-THz telescope for Earth observing
}

\author{
G. Savini \\ Optical Science Laboratory \\ Physics and Astronomy Dept. \\ University College London \\ London, England, UK \\ Email: g.savini@ucl.ac.uk \\ G.Pisano \\ Dip. di Fisica \\ Rome, Italy
}

University of Rome "La Sapienza"

\author{
P.Hargrave, P.Ade, \\ A.Shitvov, C.Tucker, I.Walker \\ Astronomy and Instrumenttion Group \\ Physics and Astronomy Dept. \\ Cardiff University \\ Wales, UK
}

\author{
J.Zhang \\ Dept. of Science and Technology \\ Anglia Ruskin University \\ Cambridge, UK
}

\begin{abstract}
We report on ongoing technology development activities in the build and testing of a refractive telescope composed of metamaterial Gradient Index (GrIn) lenses based on photolithographic meshes. This concept is tailored for Earth Observation, and more precisely limb-scanning in the sub-THz bands addressing emission lines between 50 and $190 \mathrm{GHz}$.

A single GrIn lens design which includes in-built anti-reflection coating layers, allows us to produce sufficient refraction to allow an optical design of a set of compound lenses to create the first THz range telescope fully composed of metamaterials.

First lens prototypes have been built and tested spectrally and spatially and compared to analytical models showing good agreement. Prediction of telescope performance is discussed as well as future developments.
\end{abstract}

Index Terms-THz optics, Metamaterials, Flat optics, Earth Observing

\section{INTRODUCTION}

The applications of Microwave Radiometers in the sub-THz regime for Earth Observation that have been flown in space encompass:

- Meteorology including :

- Numerical Weather Forecasting

- Medium Range Forecasting

- With aspirations to include Very Short Range and Nowcasting

- Climatology : Science, Monitoring and Composition

- Oceanographic Sciences: directly and indirectly by supporting radar altimeters

- Atmospheric Sciences : Chemical Composition and Dynamics

- Cryosphere - Sea and Land Ice and Snow

- Soil Moisture (presently limited to the lowest frequency of the microwave range).

Due to the long wavelength, radiometers are considered medium resolution instruments compared to their IR and optical counterparts. The diffraction limited aperture does result in large angular beams and carries significant volume and mass implications especially for wide swaths. The microwave capability to penetrate clouds, with critical resonances allowing sounding for temperature and humidity, provides a critical edge so that the technique remains a key baseline of any such instrument.

The key features[1] that are considered here as the baseline requirement frequencies of coverage for this technology development activity are listed in the table below and are mostly associated to temperature and humidity sounding in the $60-180 \mathrm{GHz}$ range. Looking forward, there are further features of scientific interest which include the ice-retrieval and cirrus clouds feature at $243 \mathrm{GHz}$ and the cloud ice effective radius features at 325 and $340 \mathrm{GHz}$, which encourage us to explore the feasibility of higher frequency designs to widen the possible applications.

\section{MetaTel ReQuirements}

As mentioned, the range of potential end-user applications for $\mathrm{THz}$ or sub- $\mathrm{THz}$ Earth Observation in space is large. In order to optimize the exercise of technology demonstration and make it as relevant as possible to as many applications as can make use of it, we adopt requirements and goals which are consistent with application to a moderate spatial resolution temperature and humidity sounding mission concept.

Considering a refractive, on-axis optical system with a potentially wide instantaneous field of view, allows it to be exploited by newly emerging detector array technologies. Such a combination would remove the need for an optical scanning system, and greatly increase the integration time per scene pixel.

With these considerations in mind, we present the requirements and goals for the metamaterial telescope demonstrator being developed in this programme of work. 


\begin{tabular}{|c|c|}
\hline Parameter & Requirement (Goal) and Notes \\
\hline $\begin{array}{l}\text { Frequencies of line } \\
\text { features of interest }\end{array}$ & $\begin{array}{l}55 \mathrm{GHz}-\mathrm{O}_{2} \text { temperature sounding line } \\
118 \mathrm{GHz}-\mathrm{O}_{2} \text { temperature sounding line } \\
183 \mathrm{GHz}-\mathrm{H}_{2} \mathrm{O} \text { humidity sounding line }\end{array}$ \\
\hline Frequency bandwidth & $\sim 120 \%$ or $50-195 \mathrm{GHz}$ \\
\hline Aperture & $200(300) \mathrm{mm}$ diameter \\
\hline Footprint $^{1}$ & $\begin{array}{l}28(18) \mathrm{km} @ 55 \mathrm{GHz} \\
13(8.4) \mathrm{km} @ 118 \mathrm{GHz} \\
8.2(5.4) \mathrm{km} @ 183 \mathrm{GHz}\end{array}$ \\
\hline $\begin{array}{l}\text { Focal Plane curvature } \\
\text { radius }\end{array}$ & $>650 \mathrm{~mm}$ (infinite/flat) \\
\hline $\begin{array}{l}\text { Instantaneous Field of } \\
\text { View }\end{array}$ & $\pm 10^{\circ}( \pm 20)^{\circ}$ \\
\hline Image space $F / \#$ & $<4(<3)$ \\
\hline Optical efficiency & $0.6(0.8)$ \\
\hline
\end{tabular}

TABLE I

LIST OF REQUiREMENTS/GOALS IMPOSED ON THE METATEL DESIGN.

(1) BASED ON A $817 \mathrm{KM}$ ORBIT.

\section{MetaTel telescope Design}

The range of frequencies of interest and the dimensions required are fully compatible with two demonstrated concepts of high throughput flat metamaterial lenses ([2] and [3]). The first one adopts the patterned photolithography as a means for engineered refractive index and Gradient Index (GrIn) lens design and the second using the same technology to create a phase-engineered approach which aims at moulding the phase delay radially to converge a planar input beam.

As a result, two fully viable concepts of metamaterial telescope were devised. While each concept had specific design advantages, the manufacturing burden of the full telescope is roughly equivalent and performances are fairly similar.

Due to limited time and resources, and building on prior sample data, the former of the two lenses (GrIn) was carried forward in this activity and fully developed

\section{A. Lens design principle}

In a GrIn lens design, the trade-off is between the following parameters:

- The maximum achievable index (at the centre, on axis, of the lens)

- The diameter of the desired lens

- The gradient of the index

- The thickness of the lens

The first two of these which are in tension are the highest "n" that can be emulated and placed at the centre of the lens and the lens diameter. A larger diameter "dilutes" the gradient power of the lens and therefore decreases the converging power (a simple analogy is highest index vs lens thickness, and therefore as the diameter increases, the set thickness constrains the maximum radius of curvature).

With a set converging power of the lens, the GrIn lens provides a "focusing power per unit thickness" which in turn points to a set thickness of the lens for a required focal length and working $F / \#$ needs. However, while a single very thick lens would achieve this, similarly to standard optics, a large focal plane with minimal aberrations requires a distributed focusing elements approach which is shown in Fig.1.

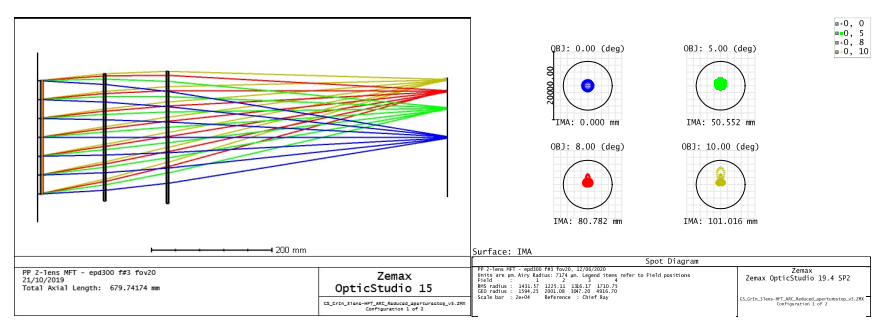

Fig. 1. A first, simple ray-tracing of the set of 3 GrIn lenses and the achievable focal plane. Rays show the $0,5,8$ and 10 degree input fields.

\section{B. Spectral performance measured}

The required Anti-Reflection-Coating (ARC) of this type of lens is added as a 2D-tapered-index layer on both sides of the lens core design, and subsequently iterated with the entire lens to account for the additional focusing power of the coating layers. The coating is optimized for the two frequencies 118 and $183 \mathrm{GHz}$. A modelled performance is shown in Fig.2.

Fig. 3 shows the measured performance of the central part of the lens. It is important to point out that the aim of the lens is to maximize throughput and efficiency of the desired spectral range. Different ring sections of the lens will have different capacitance levels and therefore act differently with their lowpass frequency cut-off (above the desired band). Nevertheless efficiency across the lens will change and therefore only the full aperture integrated spectral test can be compared to the modelling.

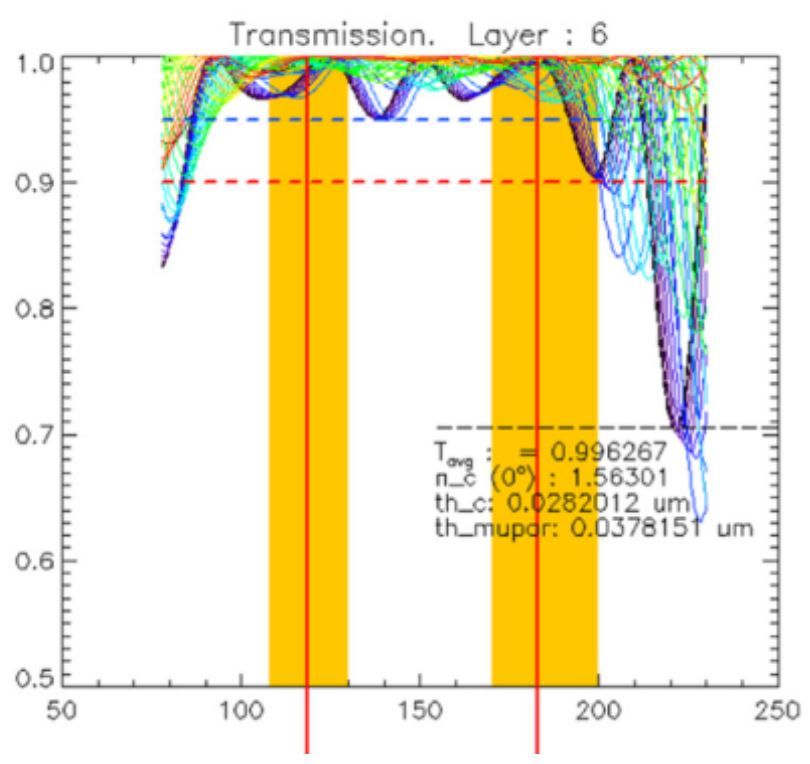

Fig. 2. Modelled spectral performance of the entire averaged lens

The contrast between expected performance (above 0.9 transmission in the range $90-200 \mathrm{GHz}$ ) and the average 


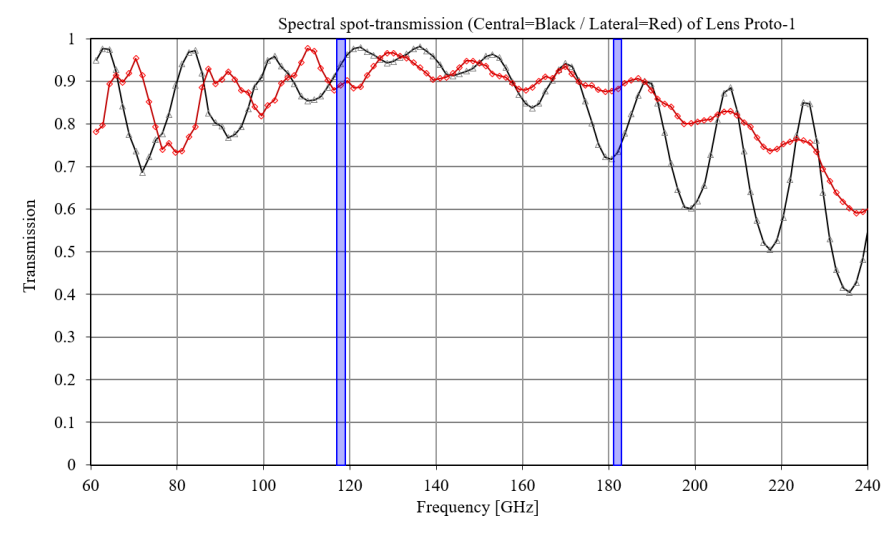

Fig. 3. Measured spot-spectral performance of the lens prototype.

performance in Fig. 3 across the lens surface (which can be estimated to be roughly 0.8 in the same spectral range, or 0.9 in a reduced range $105-155 \mathrm{GHz}$ ) can be attributed to variations in the build parameters which are under investigation, such as:

- Polypropylene index of refraction after hot-pressing (the assumed value being 1.49 and the potential resulting index being 1.51).

- A variation of thicknesses in the resulting hot-pressed prototype which result in a $7 \%$ contraction. This in turn reduces the tuning of the ARC for a less efficient performance

\section{Preliminary spatial performance (beam-slice)}

A preliminary test to verify the focusing behavior of the lens was performed with a near field scanner at a range of frequencies. The plot shown in the Fig. 4 shows the co-added beam plots for frequencies between 130 and $180 \mathrm{GHz}$.

\section{CONCLUSIONS}

We have reported preliminary performance assessment of the first prototype of a large $(200 \mathrm{~mm})$ format, low reflection GrIn lens working in a broad frequency range which acts as the main component of a three-lens telescope for Earth Observation missions. Subsequent steps will be the comparison of the three identical lenses built on the same design recipe to verify repeatibility levels of manufacturing process and how this affects lens production.

Finally, design iteration on the position of the lenses to take into account any variation in performance will allow to mitigate deviations from the intended performance of each lens when combining them to form the final telescope design.

\section{ACKNOWLEDGEMENTS}

The work presented here is funded by the UK Space Agency through the Centre for Earth Observation Instrumentation and Space Technology (CEOI-ST). For more information on the CEOI-ST activities visit www.ceoi.ac.uk .

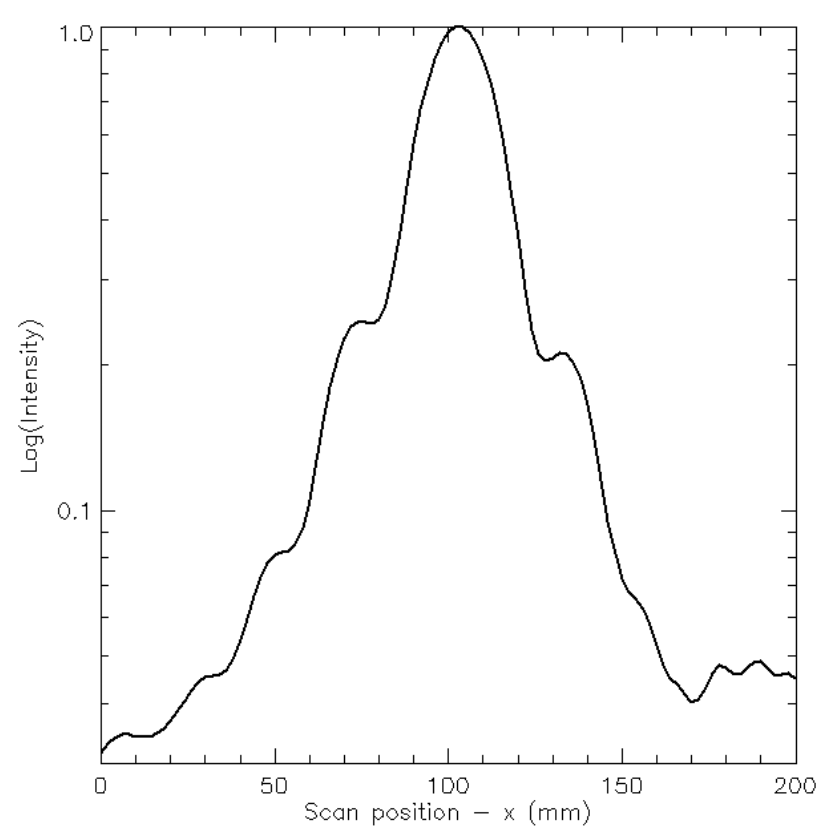

Fig. 4. Measured spectral-integrated intensity (normalized) distribution across the focus of the prototype metamaterial lens.

\section{REFERENCES}

[1] "MetOp Second Generation instruments," ESA, March 9, 2011, https://earth.esa.int/web/eoportal/satellite-missions/m/metop-sg (Accessed 25/06/2021)

[2] G.Savini, P.Ade, J.Zhang "A new artificial material approach for flat $\mathrm{THz}$ frequency lenses", Optics Express, p.25766, Vol. 20, No. 23. (2012)

[3] G.Pisano et al. "Dielectrically embedded flat mesh lens for millimeter waves applications", Applied Optics, p.2218, Vol. 52, No. 11 (2013) 